\title{
FUZZY CONTROL TECHNIQUE APPLIED TO MODIFIED MATHEMATICAL MODEL FOR MALARIA CONTROL
}

\author{
P. E. Orukpe 1 ,*and A. O. Muhammed ${ }^{2}$

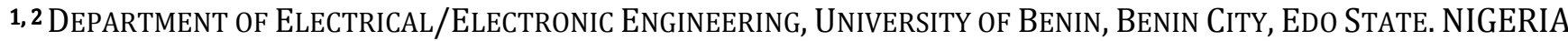 \\ Email addresses. ${ }^{1}$ patience.orukpe@uniben.edu, ${ }^{2}$ abdu4engine91@yahoo.com
}

\begin{abstract}
In this paper, fuzzy control technique is applied to the modified mathematical model for malaria control presented by the authors in an earlier study. Five Mamdani fuzzy controllers are constructed to control the input (some epidemiological parameters) to the malaria model simulated by 9 fully nonlinear ordinary differential equations (ODE) simulator of the Modified Ngwa and Shu's Model. Each of the controllers uses two different and unique variable parameters to calculate commanded control inputs to the simulator. The input domains are divided into 3 membership functions resulting in 7 fuzzy rules for each rule base and the output domains are partitioned with 5 membership functions. The Mamdani controllers use a standard max-min inference process and a fast centre of area method to calculate the crisp control signals. Numerical results obtained using Matlab 2008a software guarantee that malaria infection can be reduced to a tolerable limit.
\end{abstract}

Keywords: fuzzy control, malaria, drug effectiveness, mosquitoes, equilibrium state, dynamic equation

\section{INTRODUCTION}

Malaria is a vector borne infectious disease that has affected the human race since earliest times and an estimated $40 \%$ of the world's population lives in malaria endemic area[2]. Malaria is one of the most common infectious diseases and enormous public health problem. The yearly mortality from malaria is about 9 times that of HIV/AIDS. Malaria ranked among the major infectious disease causing deaths, after pneumococcal acute respiratory infection designated pneumonia and tuberculosis [3]. Malaria is a parasitic disease caused mainly by plasmodium falciparum. (p. falciparum) and three other malaria parasites that cause milder diseases in human (p.vivax, p.ovale, and p. malariae) are transmitted by more than a dozen species of Anopheles mosquitoes widely [4]. The disease kills about 1 to 3 million people yearly, $75 \%$ of whom are African children [5].

The incidence of malaria has been on the increase recently owing to parasite-drug-resistance and mosquito-insecticide-resistance [6]. Thus, this has lowered the productivity of the economy of the endemic zone [7]. Therefore, it is crucial to understand the important transmission parameters of the disease and develop efficient and effective solution strategies for its prevention and control.

Fuzzy control research was started by Mamdani's pioneering work in 1974, which was motivated by Zadeh's two papers on fuzzy algorithms and linguistic analysis. Fuzzy logic is a logic system that provides human reasoning capabilities to capture uncertainties, which cannot be described by precise mathematical models. It has proven to be powerful in dynamic system control. Its main merit is that it gives the most efficient knowledge representation method that can be devised for rule-based systems that deals with continuous variables [8].

Fuzzy systems have attracted the interest of researchers in various scientific and engineering areas [9]. The number and variety of applications of fuzzy logic have been increasing, ranging from consumer products [10], attitude control in spacecraft [11], and industrial process control $[13,14]$ to medical instrumentation information system and decision analysis [9] and recently to diabetic control [12]. The main aim of using fuzzy logic in medicine is the fact that medical practitioners exhibit variation in decision making as a result of their approaches to dealing with uncertainties in knowledge and information. Also, the 
diagnostic decisions depend on experience, expertise and perception of the practitioner. However, as the complexity of the system increases, it becomes necessary to use fuzzy logic as it is not easy to follow a particular path of diagnosis without any mistake. In other words, fuzzy logic renders precise what is imprecise in the world of medicine, as it is important for symptomatic diagnosis remedies.

In this paper, we apply fuzzy logic to the modified Ngwa and Shu's model presented in [1] which includes human recruitment rate, it does not restrict the total number of bites on humans only to the number of mosquitoes as the total number of bites depend on both the human and mosquito population size in our model. Likewise, we included not only the birth of susceptible mosquitoes' offsprings but also infectious offsprings of infected mosquitoes, the probability that a recovering individual become infected by one infected mosquito per contact unit time and effectiveness of drugs are included. But the model excludes direct human recovery from the infections to the susceptible class.

\section{MATHEMATICAL MODEL}

In this section, the modified mathematical model of Ngwa and Shu's model for the evolution of the malaria in a population formulated in [1] is presented in (1) (9). The equations are based on both human and mosquito population, infection rates of human and mosquito per unit time, rescaling of critical population of each class by total species population and bifurcation analysis. Following the basic ideas and structure of mathematical modeling in epidemiology, the model for the malaria disease was developed under the terms in tables 1 and 2 (see [1] for detailed analysis).

$$
\begin{aligned}
& \frac{d u}{d \tau}=\frac{\Lambda}{N_{h}}+\beta R+\lambda-\left(\alpha_{1}+\lambda+\frac{\Lambda}{N_{h}}\right) u+\gamma w u \\
& \frac{d g}{d \tau}=\alpha_{1} u-\left(v+\lambda+\frac{\Lambda}{N_{h}}\right) g+\gamma w g \\
& \frac{d w}{d \tau}=v g-\left(\gamma+\alpha_{2} A+\lambda+\frac{\Lambda}{N_{h}}\right) w+\gamma w^{2}+\alpha_{3} z N_{v} R \\
& \frac{d R}{d \tau}=\alpha_{2} A w-\left(\beta+\alpha_{3} z N_{v}+\lambda+\frac{\Lambda}{N_{h}}\right) R+\gamma w R \\
& \frac{d x}{d \tau}=a(1+(1-\theta)) z-\left(\alpha_{4}+a\right) x \\
& \frac{d y}{d \tau}=\alpha_{4} x-(a+e) y \\
& \frac{d z}{d \tau}=e y+(\theta a-a) z
\end{aligned}
$$

The equation for the total population of human and mosquitoes is now represented as:

\begin{tabular}{|c|c|}
\hline Symbol & Description \\
\hline$\Lambda_{\mathrm{h}}$ & The recruitment rate \\
\hline $\mathrm{T}$ & Time \\
\hline$\beta_{\mathrm{h}}$ & Rate at which a human looses his immunity \\
\hline$\lambda_{\mathrm{h}}$ & Per capita human birth rate \\
\hline$\lambda_{\mathrm{v}}$ & Per capita mosquito birth rate \\
\hline$\alpha_{1}$ & $\begin{array}{l}\text { The probability that a susceptible individual } \\
\text { become infected by one infected mosquito } \\
\text { per unit contact time }\end{array}$ \\
\hline$\gamma_{\mathrm{h}}$ & Infected humans who die from the disease \\
\hline$\alpha_{2}$ & The recovery rate of the infected human \\
\hline A & $\begin{array}{l}\text { The measure of the effectiveness of anti- } \\
\text { malaria drugs }\end{array}$ \\
\hline$\alpha_{3}$ & $\begin{array}{l}\text { The probability that a recovering individual } \\
\text { become infected by one infected mosquito } \\
\text { per contact unit time }\end{array}$ \\
\hline$\alpha_{4}$ & $\begin{array}{l}\text { The probability that a susceptible mosquito } \\
\text { becomes infected }\end{array}$ \\
\hline$\theta$ & $\begin{array}{l}\text { Proportion of the offspring of the infected } \\
\text { mosquito that are infected }\end{array}$ \\
\hline$(1-\theta)$ & $\begin{array}{l}\text { Proportion of the offspring of the infected } \\
\text { mosquito that are susceptible }\end{array}$ \\
\hline$\mu_{1 \mathrm{~h}}$ & $\begin{array}{l}\text { Density-independent part of the death (and } \\
\text { emigration) rate for humans }\end{array}$ \\
\hline$\mu_{2 \mathrm{~h}}$ & $\begin{array}{l}\text { Density-dependent part of the death (and } \\
\text { emigration) rate for humans }\end{array}$ \\
\hline$\mu_{1 \mathrm{v}}$ & $\begin{array}{l}\text { Density-independent part of the death (and } \\
\text { emigration) rate for mosquitoes }\end{array}$ \\
\hline$\mu_{2 \mathrm{v}}$ & $\begin{array}{l}\text { Density-dependent part of the death (and } \\
\text { emigration) rate for mosquitoes }\end{array}$ \\
\hline$w$ & $\begin{array}{l}\text { Ratio of population of infectious humans to } \\
\text { total human population }\end{array}$ \\
\hline
\end{tabular}

$$
\begin{gathered}
\frac{d N_{h}}{d \tau}=\Lambda+\lambda N_{h}-\left(\mu_{1 h}+\mu_{2 h} N_{h}\right) N_{h}-\gamma w N_{h} \\
\frac{d N_{v}}{d \tau}=a N_{v}-\left(\mu_{1 v}+\mu_{2 v} N_{v}\right) N_{v}
\end{gathered}
$$

Table 1: The State Variables for the Malaria Model

\begin{tabular}{ll}
\hline Symbol & Description \\
\hline$S_{h}$ & Population of susceptible humans \\
$E_{h}$ & Population of exposed humans \\
$I_{h}$ & Population of infectious humans \\
$R_{h}$ & Population of recovered (immune and \\
$S_{V}$ & asymptomatic, but slightly infectious) humans \\
$E_{V}$ & Population of susceptible mosquitoes \\
$I_{v}$ & Population of exposed mosquitoes \\
$\mathrm{N}_{h}$ & Population of infectious mosquitoes \\
$\mathrm{N}_{\mathrm{v}}$ & Total human population \\
\hline
\end{tabular}

Table 2: The Parameters for the Malaria Model 


\begin{tabular}{ll}
\hline Symbol & Description \\
\hline$\beta$ & $\begin{array}{l}\text { Ratio of the rate at which a human looses his } \\
\text { immunity to unit time rescale }\end{array}$ \\
$\mathrm{V}$ & Human incubation rate \\
\hline
\end{tabular}

An interesting note is that since the equations are with respect to $d \tau$, this means that the unit time measurement is with respect to the life span of a mosquito which is about 21 days [2].

\section{MODIFIED MODEL OUTPUT CONTROL}

The major task of malaria control system (model) is to keep the population in a healthy equilibrium while minimizing or reducing the infections in humans (by treatment, vaccination) and eliminating (reducing) vector populations by direct physical control and the use of insecticides within a space of time. In this work, the model is required to follow the predetermined parameter value.

Malaria dynamical model ((1)-(9)) is highly nonlinear, multivariable with significant degree of time variation due to the life span of the anopheles mosquito and change in immunity and environmental conditions. The 9-ODE model consists of 5-human compartmental variables and 4-vector compartmental variables.

The core of a malaria control system is the parameters of ODE model through which infection equilibrium point movement and control is realized and designed (e.g. $\alpha_{1}, V, \alpha_{2}, \alpha_{4}$, and $\beta$ ).

This work is focused on the 9-ODE input variable control of the modified malaria model with a Mamdani based direct control strategy as shown in Figure 1.

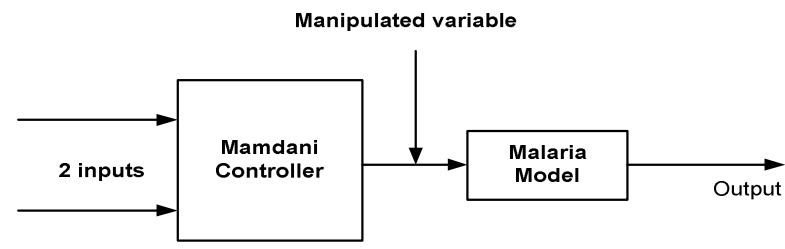

\section{Figure 1: Mamdani controller for malaria transmission control}

The two inputs to the Mamdani controller can be any of the following: Body exposure and water retention, medication and rest, infected host rise and biting rate, good diet and fitness, and dosage and tolerance. The inputs variable control requirements are described by the commanded dynamic variable, the infectious rate of human $\left(\alpha_{1}\right)$, the recovery rate of infected human $\left(\alpha_{2}\right)$, the mosquito rate of infection $\left(\alpha_{4}\right)$, the human incubation rate $(V)$, and ratio of the rate at which human looses immunity to unit time rescaled $(\beta)$, which are predetermined dynamic parameters of the modified malaria model based on the inputs to the Mamdani controller. These variables result in favourable epidemiological changes required to change the actual malaria model population to a required (stable) equilibrium states i.e. increased recovered and immunized humans and decrease infectious humans and vectors.

The infectious human, and recovered human, are controlled by a Mamdani controller with medication and food (and/rest) as inputs and the commanded $\alpha_{2}$ as output. The exposed human, $g$, and susceptible human, $u$, are controlled using degree of body exposure and water level as inputs and commanded $\alpha_{1}$ as output. The susceptible mosquitoes, $\mathrm{x}$, and exposed mosquitoes, $y$, are controlled using increase-ininfected host and biting rate as inputs and the calculated $\alpha_{4}$ as output. Also the exposed humans, g, and the infected humans, $\mathrm{w}$, are controlled using good diet and fitness as inputs and the commanded $\mathrm{V}$ as output. Finally, the susceptible human and the recovered human are controlled using dosage and tolerance as inputs and the calculated $\beta$ as output.

The input and output range used in the Mamdani is as shown in Table 3. Figure 2 shows the controller structure for one of the output state variable.

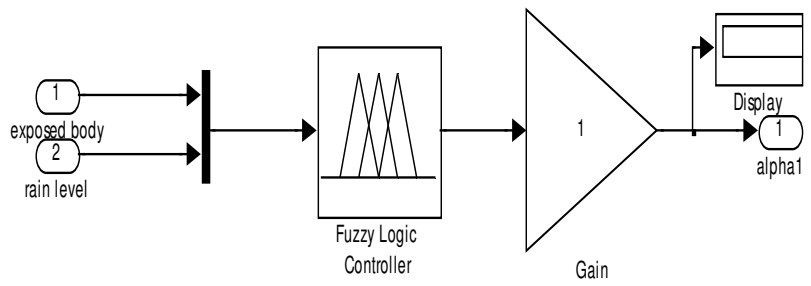

Figure 2: The structure of the Mandani controller used in the Simulink simulation environment

Table 3: Input and output- domains used in the Mamdani output state variable controller

\begin{tabular}{lll}
\hline $\begin{array}{l}\text { Para } \\
\text { met }\end{array}$ & Input range & Output range \\
er & & \\
\hline$\alpha_{1}$ & $0 \leq$ input $\leq 1$ & $0 \leq$ output $\leq 0.5$ \\
V & $0 \leq$ input $\leq 1$ & $0 \leq$ output $\leq 3$ \\
$\alpha_{4}$ & $0 \leq$ input $\leq 1$ & $0 \leq$ output $\leq 0.5$ \\
$\alpha_{2}$ & $0 \leq$ input $\leq 1$ & $0 \leq$ output $\leq 0.5$ \\
$\beta$ & $0 \leq$ input $\leq 1$ & $0 \leq$ output $\leq 0.05$ \\
\hline
\end{tabular}

\section{RESULTS AND DISCUSSIONS}

The modified malaria control model is tested in a simulation environment using the 9 ODE Simulators. The test system structure in Simulink environment is depicted in Figure 3. Figures 4-9 in which the results 
of the fuzzy based method applied to the modified mathematical model is compared with the analytical results of [1] for exposed, infectious and susceptible humans (host) and mosquitoes (vectors).

Figure 4 shows the exposed populations for humans and the results are similar except for the analytical that peak and subsequently fall. Figure 5 presents the infectious population for humans which is similar too, except for the analytical result that peak and fall subsequently. The deviation in the Figures is based on the fact that malaria cannot be reduced to zero completely as indicated by the fuzzy technique. However, the fuzzy technique gives an indication that is comparable to the analytic method for diagnosis purpose. While Figure 6 shows the susceptible population for humans and the results indicates that there is decrease in population. Similarly, the results for exposed, infectious and susceptible mosquitoes are presented in Figures 7 - 9. It is observed that they follow the same pattern with that for humans.

The peaks for the exposed population indicate movement of respective individual from the susceptible class into the exposed class. While the falls indicate that individual concerned eventually leaves the exposed class. For the infectious class, the fall is gradual in vector class than the host's. The peak shows movement of the respective individual into the infected class from the exposed class while the fall indicates that individual eventually leaves the infected class [1].

In the simulated cases using fuzzy control, it is observed that there is no peaking of any of the individuals and the fall was steep in the vector cases than the host cases.

From the results obtained, we can say that the fuzzy based method of malaria control is comparable to the analytical method.

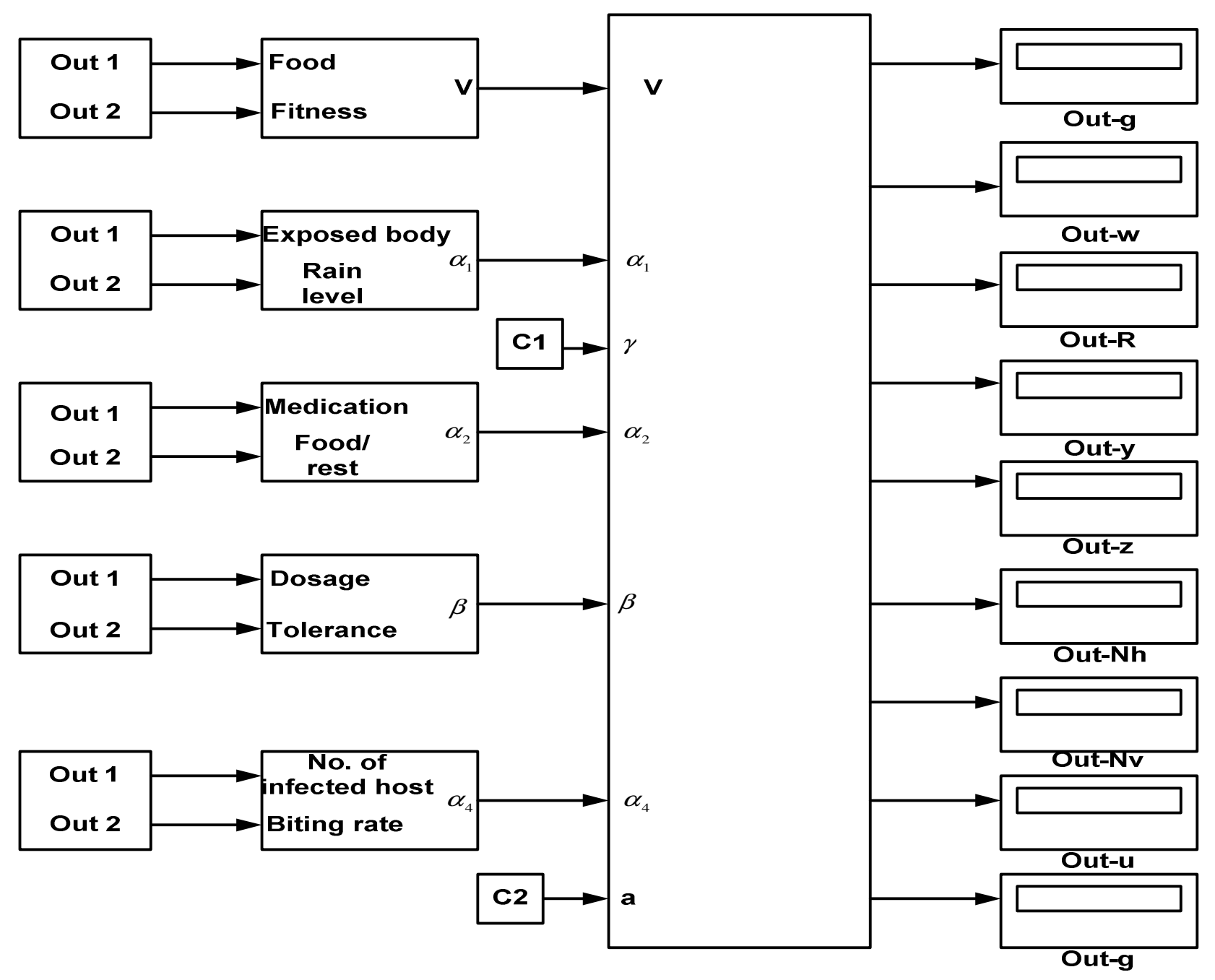

Figure 3: Simulink system structure 

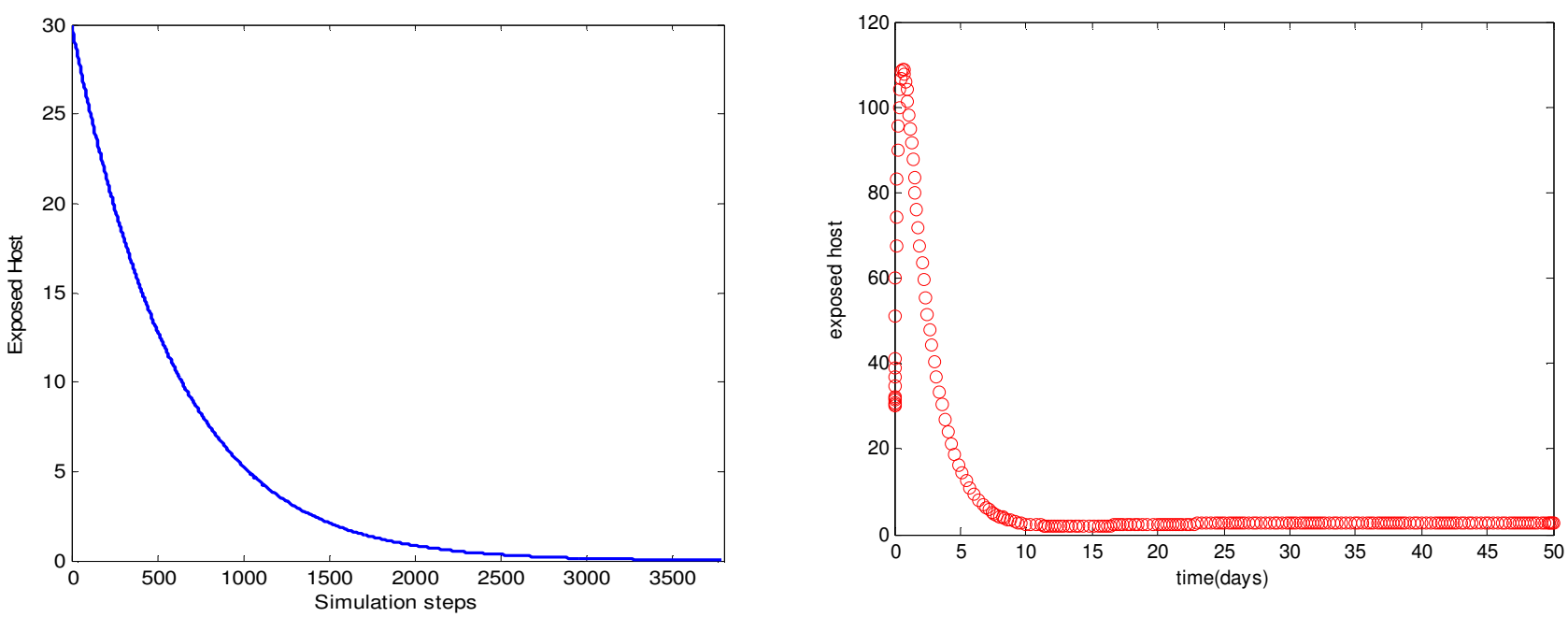

Figure 4: Exposed Host for fuzzy based method and analytical method respectively
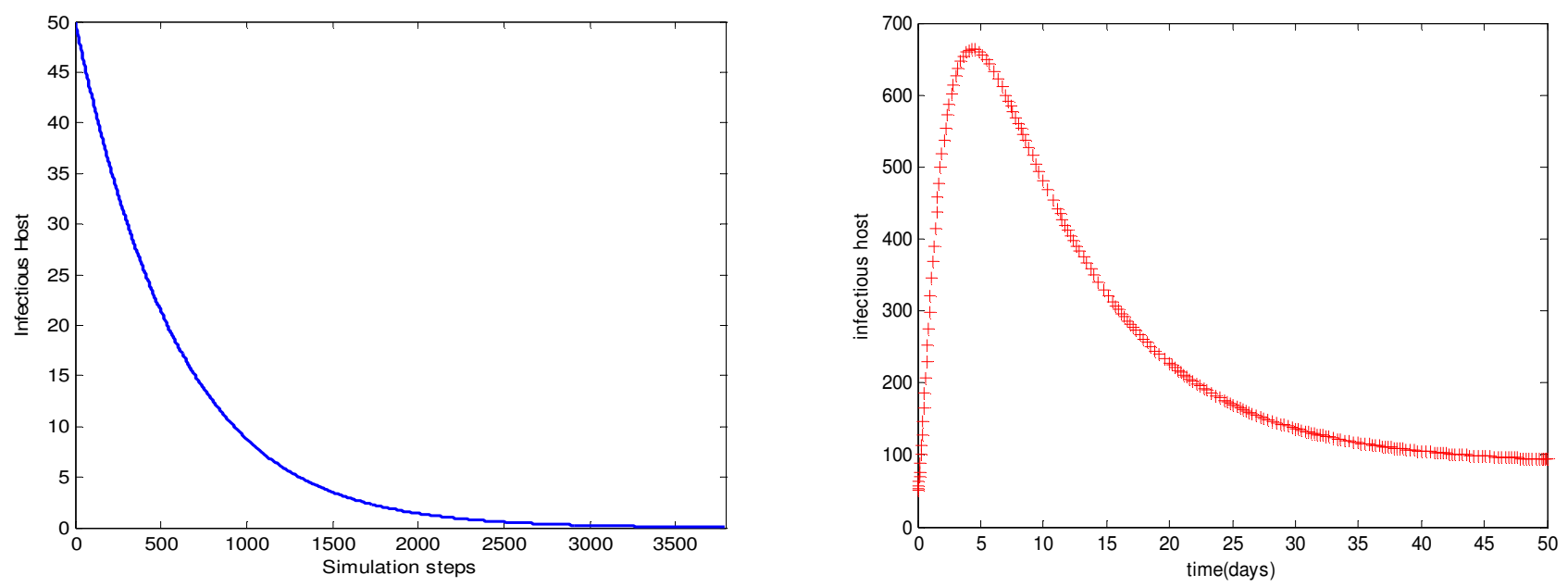

Figure 5: Infectious Host for fuzzy based method and analytical method respectively
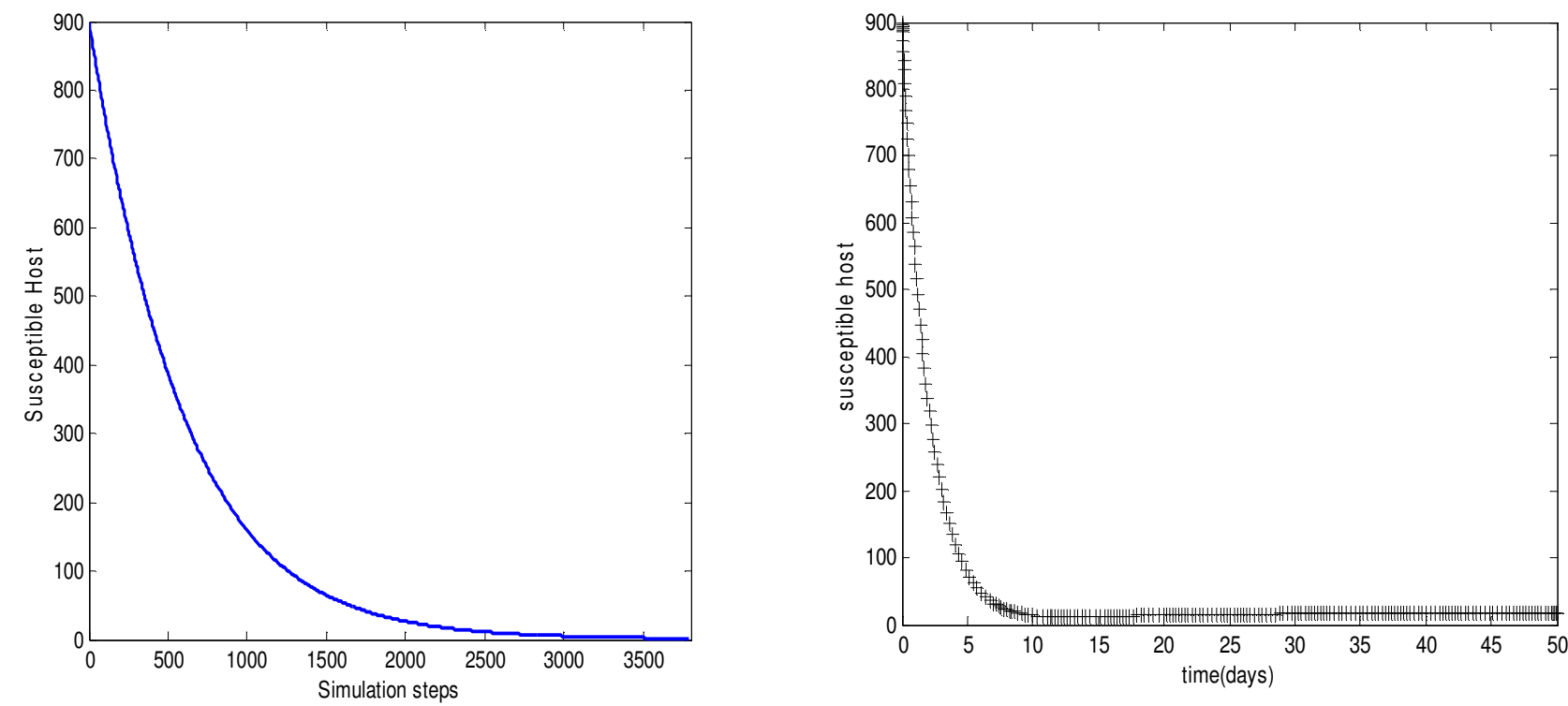

Figure 6: Susceptible Host for fuzzy based method and analytical method respectively 

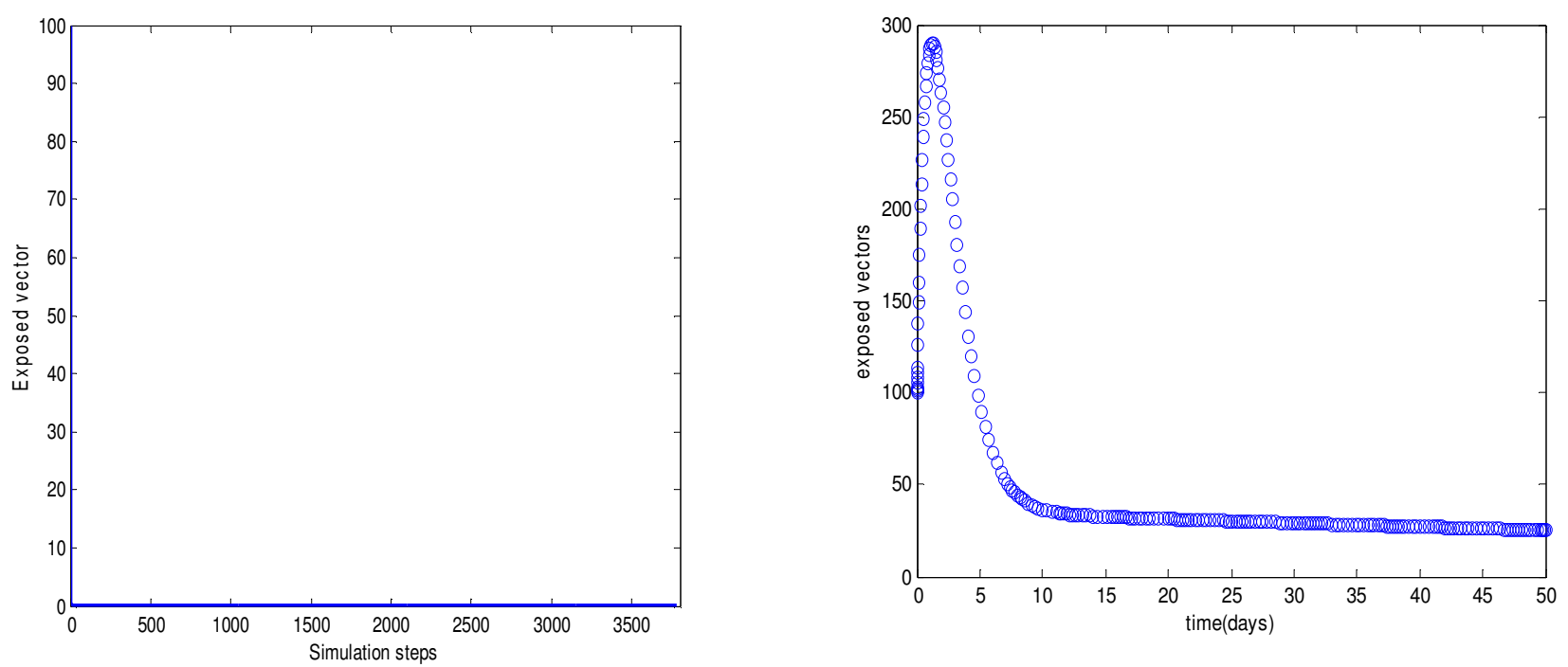

Figure 7: Exposed vector for fuzzy based method and analytical method respectively
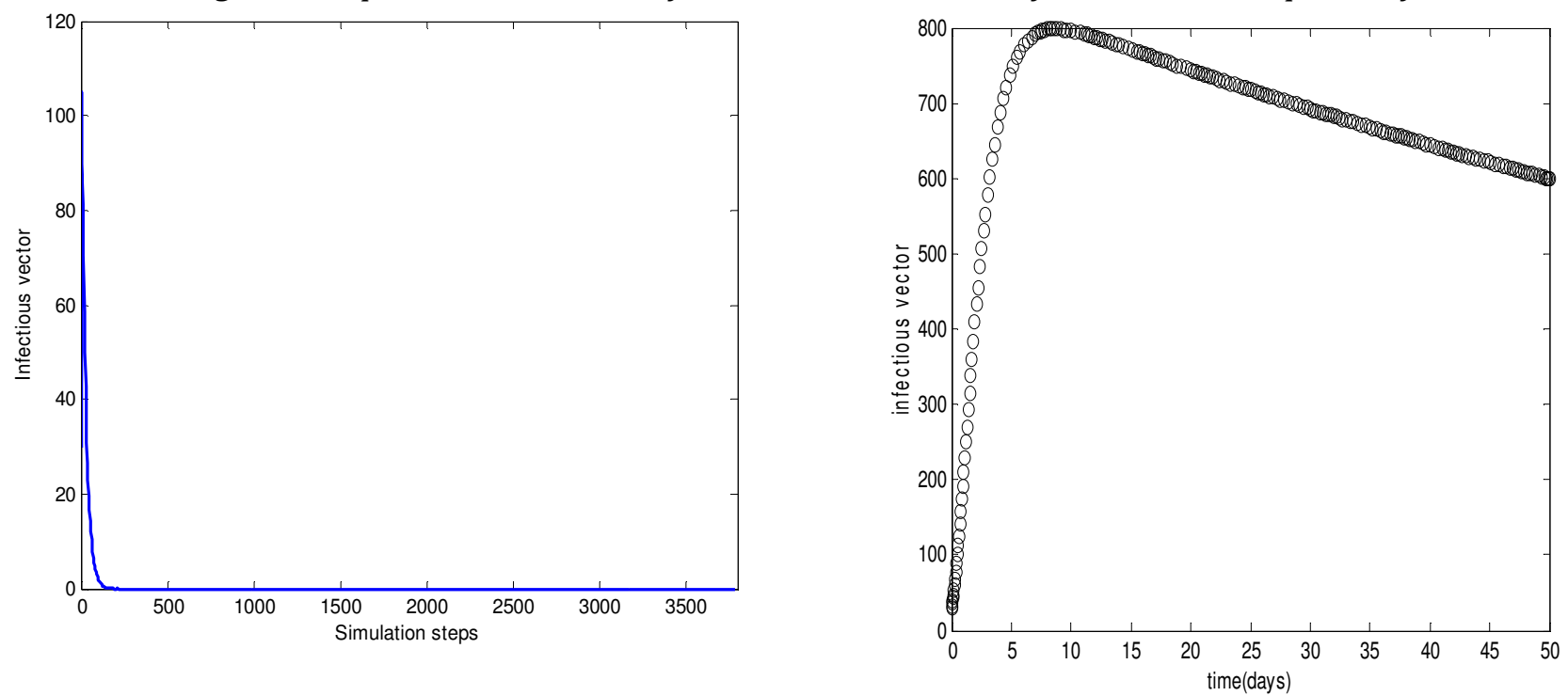

Figure 8: Infectious vector for fuzzy based method and analytical method respectively
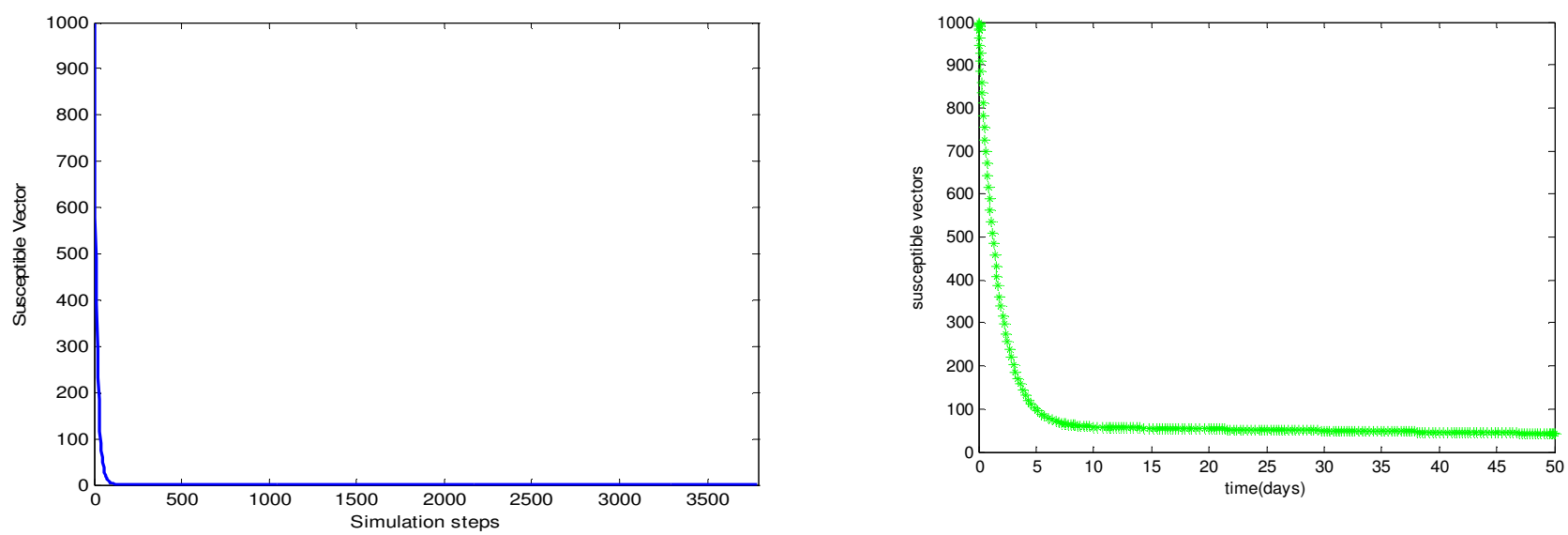

Figure 9: Susceptible vector for fuzzy based method and analytical method respectively

\section{CONCLUSION}

In this paper, fuzzy control was applied to the modified mathematical model developed for malaria control analyzed in [1]. The aim is to encourage decision making by medical practitioners using vague description for diagnosis. The fuzzy model designed is to control some of the malaria parameters in a given environment in order to have an environment where 
malaria is reduced to a tolerable limit. Tolerable limit implies that the fuzzy technique helps in reducing the infectious humans and mosquitoes population as presented in Figures 4 - 9. The results obtained shows that they are comparable with any other method.

\section{REFERENCES}

[1] Muhammed A. O. and Orukpe P. E, "Modified mathematical model for malaria control," International Journal of Applied Biomedical Engineering, Vol. 7 No. 1, pp. 1-10, 2014.

[2] W. R. Plemmons, "A mathematical study of malaria models of Ross and Ngwa", Masters Thesis, University of Central Florida, Orlando, 2006.

[3] Mohammed B. A., "Mathematical model for assessing the control of and eradication strategies for malaria in a community," Report and Opinion, 4 (2), pp. 7 $12,2012$.

[4] Pongsumpun P. and Mumtong P., "Mathematical model for the incubation of the plasmodium vivax malaria," International Journal of Applied Biomedical Engineering, Vol. 4, No. 1, pp. 42 - 48, 2011.

[5] Chitnis N., Cushing J. M., and Hyman J. M., "Bifurcation Analysis of Mathematical Model for Malaria Transmission," SIAM Journal of Applied Mathematics Vol. 67, No. 1, pp. 24 - 25, 2006.

[6] Mackinnon M. J., "Drug resistance models for malaria,” ACTA Tropica 94, pp. 207-217, 2005.
[7] Alaba O. A. and Alaba O. B., "Malaria in Rural Nigeria: Implications for the Millennium Development Goals," Africa Development Review, Vol. 21, pp. 73 - 85, 2009.

[8] Doyle D. J., Practical and Philosophical Applications of Fuzzy logic: A brief Introduction, pp. 1-10, 2012.

[9] Smita S. S., Sushil S. and Ali M. S., "Fuzzy expert systems (FES) for medical diagnosis," International Journal of Computer Applications, Vol. 63, No 11, pp. 7-16, 2013.

[10] Azar A. T., “Adaptive neuro-fuzzy systems," Fuzzy Systems, A. T. Azar (Ed.), ISBN: 978-953-7619-92-3 InTech, pp. 85-110, 2010.

[11] Okoh P. E. and Igbinovia S. O., "Attitude control of a spacecraft using fuzzy logic approach," Journal of Electrical/Electronic Engineering, Vol. 11, No. 1, pp. 1-12, 2007.

[12] Agbontaen F. O. and Orukpe P. E., "Diabetic control based on fuzzy logic," Nigerian Journal of Biomedical Engineering, Vol. 11, No. 1, pp. 42-46, 2013.

[13]. Nwodoh T. A. and Ejimofor I. E., "Implementation of fuzzy logig based- temperature controlled heat exchanger," Nigerian Journal of Technology, Vol. 29, No. 1, pp. 94-109, 2010.

[14]. Ekemezie P. N., "Evaluation of multivariable selflearning fuzzy logic controller, Nigerian Journal of Technology Vol. 22, No. 1, pp. 39-45, 2003. 